\title{
Should I Call Now? Understanding What Context is Considered When Deciding Whether to Initiate Remote Communication via Mobile Devices
}

\author{
Edward S. De Guzman, Moushumi Sharmin, and Brian P. Bailey \\ Department of Computer Science \\ University of Illinois \\ Urbana, IL 61801 \\ \{edeguzm2, sharmin2, bpbailey\}@uiuc.edu
}

\begin{abstract}
Requests for communication via mobile devices can be disruptive to the current task or social situation. To reduce the frequency of disruptive requests, one promising approach is to provide callers with cues of a receiver's context through an awareness display, allowing informed decisions of when to call. Existing displays typically provide cues based on what can be readily sensed, which may not match what is needed during the call decision process. In this paper, we report results of a four week diary study of mobile phone usage, where users recorded what context information they considered when making a call, and what information they wished others had considered when receiving a call. Our results were distilled into lessons that can be used to improve the design of awareness displays for mobile devices, e.g., show frequency of a receiver's recent communication and distance from a receiver to her phone. We discuss technologies that can enable cues indicated in these lessons to be realized within awareness displays, as well as discuss limitations of such displays and issues of privacy.
\end{abstract}

CR Categories: H.5.3 [Information Interfaces and Presentation]: Group and Organization Interfaces - collaborative computing; evaluation/methodology.

Keywords: Awareness; Diary study; Interruption; Mobile devices

\section{INTRODUCTION}

The rapid proliferation of mobile communication devices such as cell phones has enabled ubiquitous communication among family, friends, and colleagues. This has obvious benefits, but receiving requests for communication at inopportune moments can be very disruptive to the current task [33] or social situation $[2,33]$. To identify suitable moments for initiating communication, callers must be able to balance their need for communication with the receivers' need to remain engaged in the task or social situation.

Existing mechanisms for maintaining this balance include allowing receivers to choose among many notification modalities such as ring tones and ring styles (e.g., audio, vibrate, silent) or by leveraging the caller-id function. However, these mechanisms are receiver-oriented - the receiver is forced to decide whether or not to accept a call in advance without knowing the caller's purpose. Enabling callers to push more context information (e.g., text messages indicating purpose) to the receiver offers one possible solution, as it would allow a receiver to make a more informed decision of whether to accept the call. However, the explicit effort required by the caller (entering the message) and imposed on the receiver (reading and interpreting the message) may outweigh any increase in the receiver's ability to moderate incoming calls.

Graphics Interface Conference 2007

28-30 May, Montréal, Canada

Copyright held by authors. Permission granted to

CHCCS/SCDHM to publish in print form, and ACM

to publish electronically.
A promising alternative is to develop communication-oriented awareness displays that provide a caller with cues of the receiver's context (e.g., see [13, 18, 21, 34]), allowing the caller to make a more informed decision of whether now is an appropriate time to call. A compelling benefit of this approach is that it maintains the balance in effort between caller and receiver in the call initiation process. For example, a caller can consult the awareness display to decide whether to initiate the request, while a receiver may use notification modalities or caller-id to decide whether to accept.

Existing awareness displays typically provide callers with context information that can be readily sensed (e.g., presence, schedule, conversation, etc.) $[4,13,18,34]$; which may not match what is actually needed during the call initiation process. Studies confirm that callers will indeed utilize context information to coordinate their communication requests $[2,11]$, but, similarly, these studies have only considered context information that could be readily sensed or was otherwise believed to be important. As a result, there is still not adequate understanding of what range of context information should be included within awareness displays for communication devices such as mobile phones.

In this paper, we report the results of a four week diary study of mobile phone usage, where users recorded in situ what context information they considered when deciding whether to initiate a call (caller perspective), and what information they wished callers had considered before calling them (receiver perspective). Use of this particular research method allowed us to gain access to users' ongoing thought processes during everyday life situations [6]. Our study focused on the use of mobile devices due to their prevalence and because communication requests from these devices are often disruptive to our daily tasks and social situations [32].

Results from our study provide further insight into what types of context information is needed during the call initiation process. For example, our more surprising findings included that the frequency of a receiver's recent communication and distance from receiver to their phone were often considered in the call initiation process, yet are not provided in existing awareness displays.

We distill our findings into a set of design lessons that can be leveraged to improve the design of awareness displays for mobile devices. By citing existing and forthcoming technologies, we also describe realistic techniques that could be used to sense much of the context information indicated in our lessons. Finally, we present evidence from our study highlighting potential limitations of using awareness displays and user concerns of privacy.

\section{RELATED WORK}

In this section, we describe costs of interruption caused by mobile devices, techniques for coordinating communication, and how our work differs from other studies of telecommunications devices.

\subsection{Costs of Interruption}

The rapid proliferation of mobile devices has made people more accessible for communication. This offers incredible convenience,

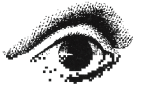


but receiving requests for communication can interrupt a person's ongoing task or social situation. Interruptions to the ongoing task can decrease performance and increase feelings of negative affect $[3,8,9,36]$. The former could be particularly serious for critical tasks such as driving $[23,33]$, while the latter may become severe enough that people will move to other physical locations [15].

Requests for communication (and the ensuing conversation) can also incur a large social cost by annoying people located near the receiver as well as by causing embarrassment to the receiver, e.g., if their mobile device rings during a lecture or ceremony [25].

Our work seeks to better understand the context information that is (or should be) considered during the call initiation process. This can lead to the design of more effective awareness displays or other techniques aimed at reducing the frequency of disruptive communication requests.

\subsection{Techniques for Coordinating Communication}

There are at least three classes of techniques for coordinating remote communication; receiver-oriented, negotiated, and calleroriented techniques. Receiver-oriented techniques are the most prevalent, and include the ability to change the notification of incoming requests to different modalities (e.g., silent, vibrate, or ring); setting modality based on caller identity; and utilizing caller identity to decide whether to accept the call [2, 29, 32]. These techniques can be useful, but shift the coordination burden onto receivers, forcing them to make decisions about communication requests in advance. This can cause important calls to be missed or unimportant calls to be accepted at inopportune moments.

A negotiated technique provides a caller and receiver with a lightweight mechanism for negotiating suitable communication times [35]. For example, if a caller requests communication at an inopportune moment, a receiver can quickly respond with a future time that would be more suitable. However, the act of negotiation itself may be disruptive to the ongoing task or social situation, as it requires at least some attention from the receiver.

Caller-oriented techniques refer mostly to awareness displays. An awareness display seeks to provide cues of a receiver's context in order to allow the caller to balance their communication needs against a receiver's context, resulting in a more informed decision of when to call. Examples include Calls.calm [28], MyVine [13], MyTeam [21], LilSys [4], AwareNex [34], and live address books [24].

An enduring challenge in building these types of displays is understanding what context information to include [16]. Most displays provide combinations of cues relating to presence, social engagement, door position, desktop interaction, location, or other easily sensed information. Though reasonable, it is not clear that these types of cues would satisfy the information needs of a caller, resulting in the displays being unused or used for unexpected purposes, e.g., to determine presence rather than availability [13]. As shown with location discourse, information need is often quite different from the information that can be readily provided [31].

Our work seeks to better understand what context information is considered during the call initiation process, thus informing the design of more effective awareness displays. This is important, as when the cues of a receiver's context match the information needs of a caller, the caller will temporally adjust their communication requests such that they occur at more opportune moments [11].

\subsection{Studies of Context and Telecommunications Devices}

In a controlled laboratory study, Avrahami et al. showed that providing callers with limited context information of a receiver can result in more appropriate interruption and better avoidance of inappropriate interruption by callers [2]. Though promising, the study considered only a limited set of context information that could be readily sensed - location of receiver, presence of other people, and the ringer status of a receiver's phone. In addition, the scenarios provided in the study were in the form of third party narratives rather than situated in daily experience. In contrast, our work leverages a diary method to elicit context information that is or should be considered during the call initiation process in situ. Our focus is on understanding what context information is needed rather than necessarily on what information could be provided.

Results from a longitudinal field study of home telephone usage show that callers and receivers come to learn each other's life rhythms, and that this influences the call initiation process [20]. For example, a caller will avoid initiating communication requests at times when s/he knows the receiver is busy, and receivers will avoid accepting requests from unknown parties during busy times, but accept requests from known parties (as it must be urgent).

As mobile devices make people more accessible, it may be difficult to understand other peoples' life rhythms to the level of detail necessary to make effective decisions of when to call. Our work thus focuses on eliciting what types of context information is considered during the call initiation process for mobile devices.

\section{DiARY STUDY}

We conducted a diary study to gain deeper insights into the types of context information that are or should be considered when deciding whether to initiate communication via mobile devices.

A diary study is a type of field study method where users record their own data in response to specific events occurring [6]. In our case, this allows the call initiation process to be studied in situ, which enables events to be examined in their natural, spontaneous context; minimizes the time between the experience of events and their capture; and eliminates the need to observe participants [6]. Diary studies have previously been used to learn more about a variety of behaviors such as computer task switching [10], making public transit decisions [7], and mobile technology adoption [26].

Though users may reflect on the call initiation process more than usual while participating in our study, we do not believe that this would prompt them to consider more, less, or different information than they would otherwise consider. Though users had to record their own data, this allowed us to gain access to their ongoing thought processes during the call initiation process.

Our study was designed to answer two main questions: (1) what types of context information does a user actually consider when

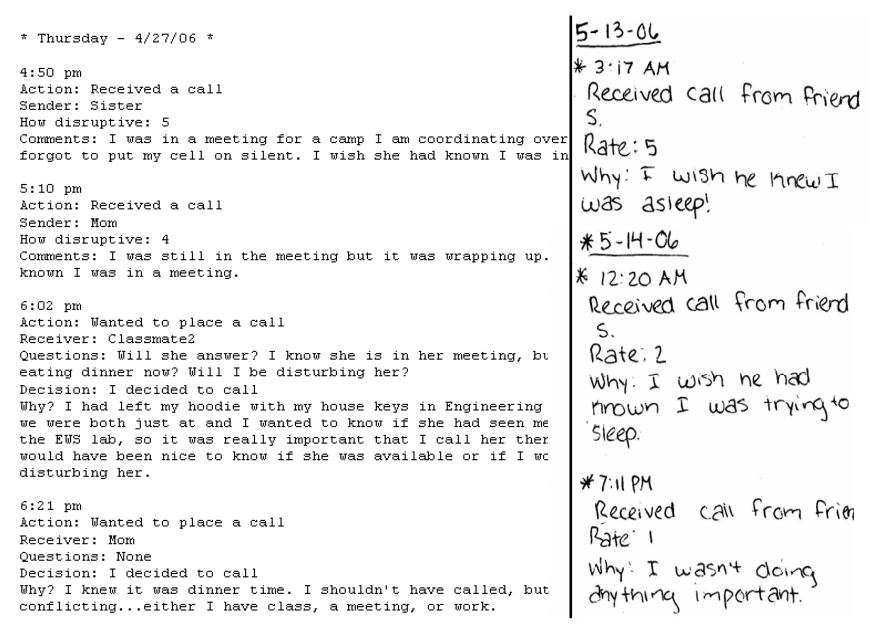

Figure 1: Sample of diary entries in electronic and paper form. 
deciding whether to initiate communication and (2) when a call is received, what types of context information does a user wish the caller had considered. Answers to these questions should help lead to the design of more effective awareness display systems.

\subsection{Users and Materials}

13 users ( 7 female, age range 19-27) participated in the study. Most were undergraduate and graduate students at our institution. Users were selected based on the criteria that they owned a mobile phone and typically used it for communication at least once a day. Users received $\$ 50$ for participating in the study.

While the age range in our study was somewhat narrow, we felt that this was not problematic as the users would likely initiate and receive calls from people of varying ages and social relationships. For example, students are likely to call people similar in age, both close friends and acquaintances; call people who are older than them (e.g., parents); and contact people outside their inner social network (e.g., secretary at an office, receptionist at a repair shop, or hostess at a restaurant). This population is also likely to receive calls from others of various ages and social relationships. Finally, people in this age range are prolific users of mobile phones.

Each user was provided with a physical diary, which was 4"x6" and weighed just a few ounces. Pages were unstructured, allowing users to enter their data in a free form manner. Users were asked to carry the diaries with them as often as possible.

\subsection{Procedure}

Each user met with the experimenter at the start of the study to go through an informed consent process, and was then provided with a diary and instructions for recording data. Any time the user made (or received) a call on their mobile phone, $\mathrm{s} /$ he was asked to make an entry into the diary just before (or after) the call.

For all entries, users were asked to include the date and time of the call and a one-word description of their relationship to the other party (mother, friend, co-worker, etc.). In addition, for calls made (caller entries), users were asked to enter a brief description of the questions they asked themselves about the context of the receiver (e.g., is the person driving?). For calls received (receiver entries), they entered a description of what they wished the caller had considered before initiating communication and rated how disruptive the call was using a 5-point Likert scale (1=not at all disruptive, $5=$ very disruptive). For both types of entries, users entered a brief justification for why the questions were asked (caller entries) or for the interruption rating given (receiver entries). Users could include any other additional details desired.

Users were asked to minimize the time between events and their recording in the diary, though several participants reported jotting down rough details of an event and filling in the remaining details at later free moments. At the end of each week, users met with the experimenter to submit their entries up to that point. Alternatively, users could transcribe and send their entries in electronic form, and many users chose this method. Sample electronic and physical entries are shown in Figure 1. The entire study lasted 4 weeks.

Table 1: Coding Agenda for the Diary Entries

\begin{tabular}{|c|c|c|}
\hline Category & Definitions & Example Entries \\
\hline $\begin{array}{l}\text { Location } \\
\qquad \text { (L) }\end{array}$ & $\begin{array}{ll}\text { Caller: } & \text { Considered the location of the receiver. } \\
\text { Receiver: } & \text { Incoming call was disruptive because of where s/he was }\end{array}$ & $\begin{array}{ll}\text { Caller: } & \text { "Is he in town?" } \\
\text { Receiver: "[l wish caller knew] I was at McKinley in } \\
\\
\text { the waiting area" }\end{array}$ \\
\hline $\begin{array}{l}\text { Time } \\
(\mathrm{T})\end{array}$ & $\begin{array}{l}\text { Caller: Wanted to know the current time before calling. } \\
\text { Receiver: Incoming call request was disruptive because of the time } \\
\text { of day }\end{array}$ & $\begin{array}{l}\text { Caller: "Will they be open at this time on Sunday?" } \\
\text { What time did he say he'd get me?" } \\
\text { Receiver: "Didn't he know it was } 3 \text { o'clock in the } \\
\text { morning!!!" }\end{array}$ \\
\hline $\begin{array}{l}\text { Physical } \\
\text { Availability } \\
\text { (PA) }\end{array}$ & $\begin{array}{l}\text { Caller: Wanted to know if there were any physical barriers to the } \\
\text { receiver being able to accept the call } \\
\text { Receiver: Physical barriers made it difficult or inconvenient for } \\
\text { receiver to answer the phone }\end{array}$ & $\begin{array}{l}\text { Caller: } \quad \text { "Is she awake?" "Will she hear my call?" } \\
\text { Receiver: "I was sound asleep"; "biking on Lincoln } \\
\\
\text { and trying to cross the street" }\end{array}$ \\
\hline $\begin{array}{l}\text { Social } \\
\text { Availability } \\
\text { (SA) }\end{array}$ & $\begin{array}{l}\text { Caller: Wanted to know if receiver was in a situation where } \\
\text { answering a call would be socially awkward } \\
\text { Receiver: Location or presence of others made it socially awkward } \\
\text { to answer the call }\end{array}$ & $\begin{array}{l}\text { Caller: "Is he with Dad?"; "Is she in ELA training?" } \\
\text { Receiver: "in a meeting and forgot to put cell on } \\
\text { silent" }\end{array}$ \\
\hline $\begin{array}{l}\text { Task } \\
\text { Status } \\
\text { (TS) }\end{array}$ & $\begin{array}{l}\text { Caller: Wanted to know if the receiver was occupied with } \\
\text { another task } \\
\text { Receiver: Was in a space suitable for receiving a call but was } \\
\text { focusing on another task }\end{array}$ & $\begin{array}{l}\text { Caller: "What is he doing?"; "Is she working?" } \\
\text { Receiver: "[I was] watching TV"; "[I was] in the middle } \\
\text { of working on homework at the Grainger } \\
\text { library }\end{array}$ \\
\hline $\begin{array}{l}\text { Emotional } \\
\text { Availability } \\
\text { (EA) }\end{array}$ & $\begin{array}{l}\text { Caller: Wanted to know if receiver was "in the mood" to talk } \\
\text { Receiver: Did not feel like talking when phone rang }\end{array}$ & $\begin{array}{ll}\text { Caller: } & \text { "Will he answer?" } \\
\text { Receiver: "I was sick and trying to sleep" "[I was] } & \text { annoyed [that] I got another call" }\end{array}$ \\
\hline $\begin{array}{l}\text { Other } \\
(0)\end{array}$ & $\begin{array}{l}\text { Caller or Receiver described questions/reasons other } \\
\text { than those mentioned above }\end{array}$ & $\begin{array}{l}\text { Caller: "Do I know what to ask?" } \\
\text { Receiver: "He just called not ten minutes ago!" }\end{array}$ \\
\hline $\begin{array}{l}\text { None } \\
(\mathrm{N})\end{array}$ & $\begin{array}{ll}\text { Caller: } & \text { Did not ask any questions as part of the call process. } \\
\text { Receiver: } & \text { This category is not applicable for receiver entries. All } \\
& \text { receivers gave a reason for their disruption rating }\end{array}$ & $\begin{array}{l}\text { "Didn't really consider anything, just called } \\
\text { him back."; "I was locked out [of the } \\
\text { apartment]" }\end{array}$ \\
\hline
\end{tabular}


Caller Entries

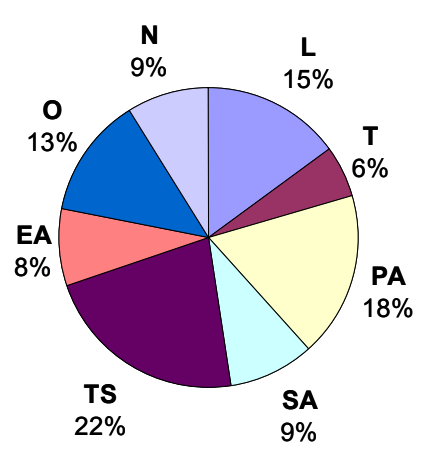

Figure 2: Frequency of context citations for caller and receiver entries.

\subsection{Coding}

A set of categories was needed to code the collected entries. Since the entries contained mostly context information, we started with the categorization proposed in [12] - location, time, activity, and identity - and adapted it in two ways. First, we removed the identity category since callers obviously know who they intend to call and receivers can assume that the caller knows who they are.

Second, we decided to further decompose the activity category, since a large proportion of the entries referenced it. Analysis of the relevant entries yielded four subcategories of activity (named relative to a receiver's availability): physical availability $(P A)$, social availability (SA), emotional availability (EA), and task status (TS). Our final agenda is given in Table 1, which includes definitions for each context category and numerous examples drawn from the actual pool of diary entries.

\section{Results}

A total of 616 entries were collected; 288 caller- and 328 receiverentries, and coded according to Table 1 . Using the same agenda, a second researcher independently coded $10 \%$ of entries (32 caller, 32 receiver), and there was $88 \%$ agreement. Differences were resolved via discussion and changes were re-applied to all entries. If a diary entry spanned more than one category, it was counted multiple times. For example, an entry including "is he home yet" and "what time is it" would be counted as Location and Time.

Overall, the 288 caller entries cited 504 instances of context information (1.75 instances per call made), while the 328 receiver entries cited 354 instances (1.08 instances per call received). This result confirms that context is (and needs to be) considered when initiating a call, and that providing just a few cues of a receiver's context could lead to more informed decisions of when to call.

\subsection{Caller and Receiver Context Citations}

The distribution of context citations for caller and receiver entries is shown in Figure 2. By far, the activity subcategories (PA, SA, $\mathrm{TS}$, and EA) were the most prominent, comprising $58 \%$ of citations in caller entries and $71 \%$ of citations in receiver entries. From the perspective of callers, the data is interesting because it shows that a caller considers a receiver's task $(22 \%)$ and physical availability $(18 \%)$ more than his/her location $(15 \%)$ and social availability $(9 \%)$.

From the perspective of receivers, the data is similar in that it shows that receivers want callers to consider their task (34\%) and

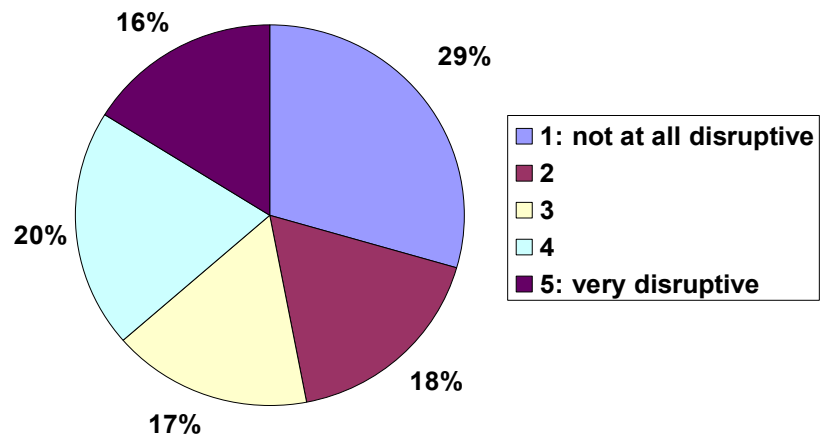

Figure 3: Frequency distribution of interruption ratings.

physical availability (21\%) more than other context information, but that receivers want callers to consider this information more often ( $34 \%$ vs. $22 \%$; $21 \%$ vs. $18 \%$, respectively). Furthermore, receivers want callers to consider their social availability more (15\% vs. $9 \%)$ and were less concerned about callers considering location (only $9 \%$ of receiver entries cited location vs. $15 \%$ of caller entries).

In addition to these common categories, our study also revealed other types of context information that are important during the call initiation process, categorized in the "Other" category. First, many caller entries cited their own context, e.g., how much free time did they have ("When do I have to leave for class?"), were others around them, and were they clear about the purpose for making the call ("Do I know what to ask?"). Second, callers also wanted to know about their past interactions with a receiver. For example, one caller wanted to know how long it has been since she last contacted a particular friend ("When did I last call her?"), whereas another caller wanted to know how many times he had called the receiver that day. Conversely, one receiver wished the caller knew that she had received four calls in the past hour and thus did not feel like taking another. This suggests that past phone interactions on both caller and receiver ends can play a role in the decision process. Finally, callers and receivers often wanted to know the distance from receivers to their phone. For example, callers stated that it was pointless to call if the receiver could not hear the phone (and did not want to leave a message), while receivers stated that it was disruptive to have to race in from outside, suspend their shower, or walk from another part of the house to answer the phone.

In our analysis, we also examined the impact of social relationship in the call decision process. Each entry was categorized by the relationship between the two parties involved. "Family/Friends" consisted of entries describing events involving immediate and extended family, as well as friends within the participant's social network. "Non-Family/Friends" entries included calls to co-workers, team members from group projects, and non-acquaintances (e.g., office receptionists, customer service representatives). Separating the caller and receiver entries based on the social relationship between the participant and the other party revealed interesting differences in the context distribution.

Relative to "Non-Family/Friends", entries in "Family/Friends" cited the other party's location (16\% of entries vs. $7 \%$ ), physical availability (19\% vs. $11 \%)$, and task status $(24 \%$ vs. $9 \%)$ more often. In the "Non-Family/Friends" entries, context coded as 


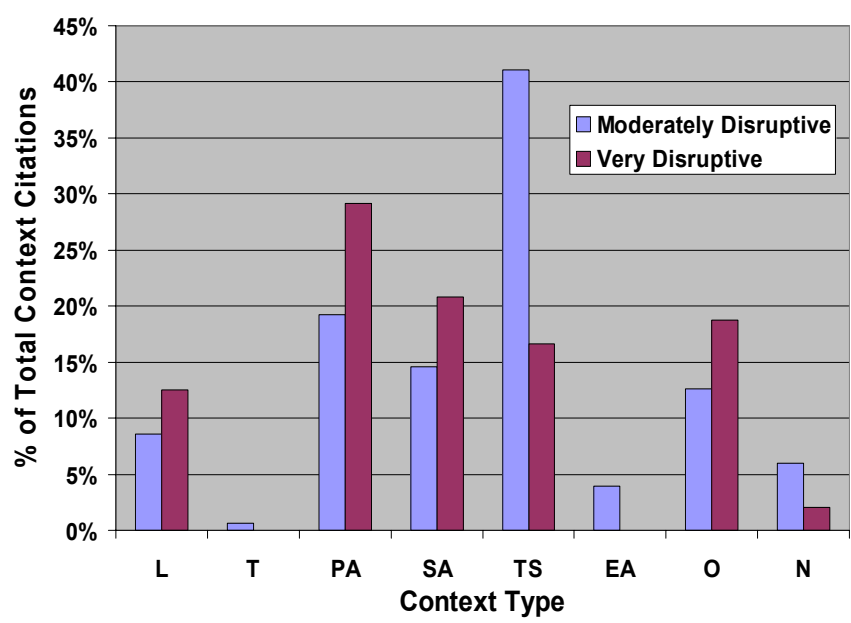

Figure 4: Frequency of context citations in receiver entries between moderately and very disruptive incoming calls.

"Other" was considered more often (42\% vs. 9\%). These findings suggest that awareness displays would be most utilized for calls initiated between Family/Friends, but further examination of how the utilization of context information might depend on the social relationship between the caller and receiver is needed.

\subsection{Interruption Ratings}

Figure 3 shows the distribution of interruption ratings for the receiver entries. $55 \%$ of calls received were deemed moderately disruptive $(1<$ rating $<=4)$ while $16 \%$ were considered very disruptive (rating $=5$ ), indicating a need for better coordination. To explore this further, we wanted to understand whether the context information that receivers wished callers had considered was different between moderate vs. very disruptive calls. Since entries corresponding to calls that were considered not disruptive (rating=1) cited little context, that data was not included here.

Figure 4 shows the distribution of context citations between the moderate and very disruptive calls. Entries rated (2, 3, or 4) were categorized as "moderately disruptive" while entries rated 5 were categorized as "very disruptive". This categorization allowed us to isolate those calls rated with the highest perceptual significance. Different categorizations, for example comparing entries with ratings of $(2,3)$ vs. $(4,5)$, produced results similar to Figure 4.

Relative to moderate, for calls deemed to be very disruptive, receivers wished that callers had given more consideration to physical availability, social availability, and other context (e.g., distance to phone and frequency of recent interaction). Focusing on these types of cues within an awareness display system would thus yield the most benefit, as it would enable and encourage callers to consider context that receivers deem important for better coordinating communication requests that would otherwise be considered very disruptive.

\section{IMPLICATIONS FOR AWARENESS DISPLAY SYSTEMS}

Overall, our study indicates that the call initiation process would benefit from an awareness display system. For example, it would give callers access to more accurate context information that they already consider (e.g., task status and physical availability) and encourage callers to consider additional context that they consider less often, but receivers deem important (e.g., social availability).

We also learned several important lessons on how to design such a system. While several of our lessons reinforce findings from previous studies, we also propose incorporating types of context not previously considered in other mobile awareness display systems. In this section, we present our lessons learned and motivate them with results from our study. Where appropriate, we suggest existing or forthcoming technology that would allow these lessons to be realized within an awareness display system.

L1. Provide cues related to a receiver's physical availability. A caller's lack of awareness of the receiver's physical availability accounted for the highest proportion of incoming calls that were rated "very disruptive". The most common reasons cited were that the receiver was sleeping or her current physical activity (e.g., driving or riding a bike) made the phone difficult or dangerous to reach. Though challenging, there are several mechanisms that could be leveraged to provide this type of context information. For example, there are small, lightweight sensors that can be safely attached to one's body in order to sense orientation [1]. Also, a receiver's motion can be computed using GPS technology [19]. A recent study of providing motion information via mobile phones showed that callers used this information to infer location and activity and to better coordinate communication [5].

L2. Show the distance from the receiver to the phone. Receivers were often frustrated when they had to walk across the apartment, suspend their shower, or race in from outside to answer the phone, as they did not always carry or have their devices near them. Though receivers could ignore the request, the fact is that people almost always attempt to answer their phone [20]. Similarly, callers may not attempt to initiate communication if they know that a receiver is not proximal to her phone. Interestingly, recent research shows that simple variables can be leveraged to predict a person's distance to their phone, with accuracy of over $85 \%$ [27], and this prediction could be conveyed via an awareness display.

L3. Inform the caller of a receiver's social availability. Receivers were often frustrated when receiving calls during group meetings or while in public spaces such as a movie theatre or concert, and often forgot to change the notification modality on their phone. One method for detecting the presence of others is to periodically capture short bursts of audio using an open microphone on their device [13]. Computational techniques exist for differentiating speech from non-speech audio and processing the data in a way that preserves the privacy of the receiver and those around them [14]. This information, combined with calendar information [18], could provide cues of social availability. Alternatively, Marti and Schmandt have explored the use of body-worn sensor nodes to determine if a receiver is actively engaged in a conversation [22].

L4. Provide more than location. Though our results show that both callers and receivers consider location, it is considered much less than other categories. For example, if a receiver is engaged in face-to-face conversation, results from our study indicate that it is more important to make the caller aware of the conversation itself than its location. Thus, though awareness displays should display location-based context, they should not rely on this alone.

L5. Provide the caller with the receiver's task status. For both caller and receiver entries, the most common context category referenced was related to task status. Callers asked questions of varying specificity, ranging from "What is he doing?" to "Is she eating dinner?" After a disruptive incoming call, receivers wished the caller knew more about their current task such as "writing a paper," "watching favorite TV program," or just "I was busy". 
Instrumentation of a receiver's computer (e.g., to sense any recent interaction [4]) and even their television (what channel it is on), combined with some of the capture methods described previously, could provide a sense of a receiver's task status. For example, if the caller was able to know that the receiver was at the library, surrounded by little audio activity, and running a document editor, $\mathrm{s} /$ he could likely infer that the receiver was writing a paper.

L6. Allow callers to consider their own context. In addition to the context of the receiver, callers sometimes asked questions about their own context. For example, callers considered whether other people were around them, how much free time they had available, and if they were clear about their own purpose of making the call. While callers themselves are best at inferring the majority of their own context, lightweight access to personal calendar information could help them decide how much time is available for a call.

L7. Provide access to a history of past interactions between the caller and receiver. Numerous entries suggested that past phone interactions between caller and receiver can impact the decision of whether to call. For example, one caller wanted to know the last time she had called her friend to avoid being a nuisance. Another caller wanted to know how many times he had called the receiver that day. Conversely, one receiver wished the caller knew that she had received four calls from other people in the past hour and thus did not feel like taking another. Most of this information can be derived from the call memory of mobile devices, but needs to be conveyed though cues that can be quickly and accurately assessed.

L8. Consider granularity when collecting and presenting receiver context. When describing context information in the diary study, the granularity varied depending on the situation. For example, when inquiring about a receiver's task status, some callers asked "Is he studying?" while others asked "Is he writing a paper?" In the first case, showing the receiver's location (e.g., at the library or in a classroom) may be sufficient. However, the second case would require more detail such as what application is active on his desktop. An awareness display should thus be able to present varying levels of detail regarding the receiver's context, but this should be a function of the identity or relationship of the person inquiring, among other relevant information [24]. Furthermore, the receiver should be able to override any default settings to disclose more or less detail than what the default setting provides.

L9. Empower callers to make inferences based on multiple cues of a receiver's context. Even as sensing technology becomes more accurate and capable of sensing more behavioral acts, there may always be a large gap between the low-level information that can be sensed and the high-level task or social situation of a receiver. Based on our results and experience gained from this study, we learned that awareness displays should be designed such that they provide callers with discrete cues of a receiver's context, rather than trying to compute a single, holistic measure of "availability." This would allow the caller to integrate the given cues with their own understanding of the receiver's life rhythms to make an informed decision of whether to initiate communication.

\section{DisCuSSION}

A goal of our work was to help systems designers understand how to develop more effective awareness displays for mobile devices. Based on in situ data collected from a four week diary study of the call initiation process for mobile devices, we have provided several lessons about what type of context information to include and how to convey that information within an awareness display.
Our lessons are most applicable to the age range studied, but an awareness display consistent with these lessons would likely be beneficial to a broader range of users, as they would ostensibly consider similar information during the call initiation process.

Awareness displays, if designed effectively, will likely improve coordination of communication requests [2], but it is important to point out that such displays are not a panacea. For example, even if an awareness display could accurately convey the context of a receiver to the caller, it may not capture the receiver's desirability of receiving a communication request. In the diary study, for example, there were many instances where a receiver rated an incoming call as "not disruptive" even though s/he was engaged in an ongoing task or social situation. In these cases, the call was welcome as it provided a needed break from the current task or a convenient means of getting away from an undesirable social situation. For example, one user was writing a paper when she received a call but was "stuck" and appreciated the call as it forced her to take a needed mental break. Also, even if an awareness display system was capable of providing callers with all of the relevant cues of receivers' context, the caller is still ultimately responsible for appropriately weighing his/her need for communication against the receiver's need to remain focused on the current task or social situation.

There were also instances suggesting that receivers may not always wish to present cues of their current status. For example, a receiver noted that though he was awakened by a call, he did not find the call disruptive because it was time to get up anyway and he did not want the caller to know he was asleep. Recent research shows that receivers are generally willing to share context [17], but this example highlights the need to ensure that receivers are able to control the level of disclosure of sensed cues. As argued in L8, allowing the receiver to explicitly control the granularity of cues disclosed is part of the solution, but receivers should also be able to specify disclosure as a function of the person inquiring, the receiver's location, and the time of day [30].

We acknowledge that building any awareness display capable of providing cues of receivers' context, along with controls over privacy, would be difficult. We thus believe that our lessons (L1L9) provide a meaningful contribution, as they will help designers focus implementation effort where it is most needed, and preclude efforts seeking to convey cues that may in fact be unnecessary.

There are some limitations to the diary study that we conducted. First, all of the study participants were living in the United States. Studying similar populations in Europe or Asia may potentially yield different results. Second, participants tended to create entries only in situations where an actual communication session took place. As a result, we were unable to analyze what types of context contributed most to a caller deciding not to make a call or a receiver deciding not to answer a call. Despite these limitations, we feel our study was successful in enabling us to collect a large data set with high ecological validity. Finally, a user in our study recorded data in a dual role, as both a caller and a receiver. We did not study differences between context information that a caller considered and that a receiver wished had been considered for specific instances of calls, or as a function of the social relationship between specific pairs of callers/receivers. Our goal was to understand the general range of context information that is considered by callers and receivers, which could be leveraged to improve the design of awareness displays for mobile devices.

\section{CONCLUSION AND FUTURE WORK}

The rapid proliferation of mobile phones allows people to be more accessible, but receiving communication requests at inopportune moments can disrupt the current task or social situation. A very 
promising approach for reducing the frequency of disruptive calls is to provide callers with cues of a receiver's context through an awareness display. Such a display would allow a caller to make a more informed decision of whether now is an appropriate time to call. A fundamental challenge is to understand just what types of context information should be conveyed in these displays. Our work has made several contributions addressing this challenge.

First, based on results from a four week diary study of mobile phone usage, our work has contributed some of the first in situ data detailing what context information users actually consider and what information they wished others had considered during the call initiation process. Second, we distilled our results into lessons that can guide the design of awareness displays and cited existing or forthcoming technology developments that could be leveraged to realize our lessons in practice. Finally, we discussed potential limitations of awareness displays in the call initiation process and highlighted the need for privacy controls.

Our future work consists of three main steps. First, we want to employ sensors and other lightweight mechanisms for collecting context information consistent with the lessons from our study. Second, we want to investigate how to best visualize cues of a receiver's context information on a small screen device. Finally, once reasonably implemented, we want to understand how the use of such an awareness display impacts the call initiation process with mobile and other communication devices.

\section{ACKNOWLEDGEMENTS}

We thank the users who participated in our study and members of our research group for commenting on earlier drafts of this article. This research was supported in part by a grant from the National Science Foundation under award no. IIS 05-34462.

\section{REFERENCES}

[1] Addlesee, M., R. Curwen, S. Hodges, J. Newman, P. Steggles, A. Ward and A. Hopper. Implementing a Sentient Computing System. IEEE Computer Magazine, 34 (8), 50$56,2001$.

[2] Avrahami, D., D. Gergle, S.E. Hudson and S. Kiesler. Improving the Match between Callers and Receivers: A Study on the Effect of Contextual Information on Cell Phone Interruptions. Behaviour and Information Technology, in press, 2007.

[3] Bailey, B.P. and J.A. Konstan. On the Need for Attention Aware Systems: Measuring Effects of Interruption on Task Performance, Error Rate, and Affective State. Journal of Computers in Human Behavior, 22 (4), 709-732, 2006.

[4] Begole, J.B., N.E. Matsakis and J.C. Tang. Lilsys: Sensing Unavailability. Proceedings of the ACM Conference on Computer Supported Cooperative Work, 2004, 511-514.

[5] Bentley, F. and C. Metcalf. Sharing Motion Information with Close Family and Friends. Proceedings of the ACM Conference on Human Factors in Computing Systems, 2007.

[6] Bolger, N., A. Davis and E. Rafaeli. Diary Methods: Capturing Life as It Is Lived. Annual Review of Psychology, 54, 579-616, 2003.

[7] Carter, S. and J. Mankoff. When Participants Do the Capturing: The Role of Media in Diary Studies. Proceedings of the ACM Conference on Human Factors in Computing Systems, 2005, 899-908.

[8] Czerwinski, M., E. Cutrell and E. Horvitz. Instant Messaging and Interruption: Influence of Task Type on Performance.
Annual Conference of the Human Factors and Ergonomics Society of Australia (OZCHI), Sydney, Australia, 2000, 356361.

[9] Czerwinski, M., E. Cutrell and E. Horvitz. Instant Messaging: Effects of Relevance and Timing. People and Computers XIV: Proceedings of HCI, 2000, 71-76.

[10] Czerwinski, M., E. Horvitz and S. Wilhite. A Diary Study of Task Switching and Interruptions. Proceedings of the ACM Conference on Human Factors in Computing Systems, 2004, 175-182.

[11] Dabbish, L. and R.E. Kraut. Controlling Interruptions: Awareness Displays and Social Motivation for Coordination. Proceedings of the ACM Conference on Computer Supported Cooperative Work, 2004, 182-191.

[12] Dey, A.K., D. Salber and G.D. Abowd. A Conceptual Framework and a Toolkit for Supporting the Rapid Prototyping of Context-Aware Applications HumanComputer Interaction, 16 (2-4), 97-166, 2001.

[13] Fogarty, J., J. Lai and J. Christensen. Presence Versus Availability: The Design and Evaluation of a Context-Aware Communication Client. International Journal of HumanComputer Studies, 61 (3), 299-317, 2004.

[14] Horvitz, E., P. Koch, C.M. Kadie and A. Jacobs. Coordinate: Probabilistic Forecasting of Presence and Availability. Eighteenth Conference on Uncertainty and Artificial Intelligence, Edmonton, Alberta, 2002, 224-233.

[15] Hudson, J.M., J. Christensen, W.A. Kellogg and T. Erickson. "I'd Be Overwhelmed, but It's Just One More Thing to Do": Availability and Interruption in Research Management. Proceedings of the ACM Conference on Human Factors in Computing Systems, 2002, 97-104.

[16] Hudson, S.E. and I. Smith. Techniques for Addressing Fundamental Privacy and Disruption Tradeoffs in Awareness Support Systems. Proceedings of the ACM Conference on Computer Supported Cooperative Work, 1996, 248-257.

[17] Khalil, A. and K. Connelly. Context-Aware Telephony: Privacy Preferences and Sharing Patterns. Proceedings of the ACM Conference on Computer Supported Cooperative Work, 2006, 469-478.

[18] Khalil, A. and K. Connelly. Improving Cell Phone Awareness by Using Calendar Information. Proceedings of INTERACT, 2005, 588-600.

[19] Laasonen, K., M. Raento and H. Toivonen. Adaptive onDevice Location Recognition. Pervasive, 2004, 287-304.

[20] Lacohee, H. and B. Anderson. Interacting with the Telephone. International Journal of Human Computer Studies, 54 (5), 665-699, 2001.

[21] Lai, J., S. Yoshihama, T. Bridgman, M. Podlaseck, P. Chou and D. Wong. MyTeam: Availability Awareness through the Use of Sensor Data. Proceedings of the IFIP Conference on Human-Computer Interaction (INTERACT), 2003.

[22] Marti, S. and C. Schmandt. Giving the Caller the Finger: Collaborative Responsibility for Cellphone Interruptions. Proceedings of the ACM Conference on Human Factors in Computing Systems, 2005, 1633-1636.

[23] McFarlane, D.C. and K.A. Latorella. The Scope and Importance of Human Interruption in HCI Design. HumanComputer Interaction, 17 (1), 1-61, 2002. 
[24] Milewski, A.E. and T.M. Smith. Providing Presence Cues to Telephone Users. Proceedings of the ACM Conference on Computer Supported Cooperative Work, 2000, 89-96.

[25] Nelson, L., S. Bly and T. Sokoler. Quiet Calls: Talking Silently on Mobile Phones. Proceedings of the ACM Conference on Human Factors in Computing Systems, 2001, 174-181.

[26] Palen, L. and M. Salzman. Voice-Mail Diary Studies for Naturalistic Data Capture under Mobile Conditions. Proceedings of the ACM Conference on Computer Supported Cooperative Work, 2002, 87-95.

[27] Patel, S.N., J.A. Kientz, G.R. Hayes, S. Bhat and G.D. Abowd. Farther Than You May Think: An Empirical Investigation of the Proximity of Users to Their Mobile Phones. Proceedings of Ubicomp, 2006, 123-140.

[28] Pedersen, E.R. Calls.Calm: Enabling Caller and Callee to Collaborate. Proceedings of the ACM Conference on Human Factors in Computing Systems, 2001, 235-236.

[29] Pering, C. Taming of the Ring: Context Specific Social Mediation for Communication Devices. Proceedings of the ACM Conference on Human Factors in Computing Systems, 2002, 712-713.

[30] Price, B.A., K. Adam and B. Nuseibeh. Keeping Ubiquitous Computing to Yourself: A Practical Model for User Control of Privacy. International Journal of Human-Computer Studies, 63 (1-2), 228-253, 2005.

[31] Reilly, D.F., D. Dearman, V. Ha, I. Smith and K. Inkpen. "Need to Know": Examining Information Need in Location Discourse. Pervasive, 2006, 33-49.

[32] Sadler, K., T. Robertson and M. Kan. "It's Always There, It's Always on": Australian Freelancer's Management of Availability Using Mobile Technologies. Mobile HCI, 2006.

[33] Schneider, M. and S. Kiesler. Calling While Driving: Effects of Providing Remote Traffic Context. Proceeding of the ACM Conference on Human Factors in Computing Systems, 2005, 561-569.

[34] Tang, J.C., N. Yankelovich, J. Begole, M.V. Kleek, F. Li and J. Bhalodia. Connexus to Awarenex: Extending Awareness to Mobile Users. Proceedings of the ACM Conference on Human Factors in Computing Systems, 2001, 221-228.

[35] Wiberg, M. and S. Whittaker. Managing Availability: Supporting Lightweight Negotiations to Handle Interruptions. ACM Transactions on Computer Human Interaction, 12 (4), 356-387, 2005.

[36] Zijlstra, F.R.H., R.A. Roe, A.B. Leonora and I. Krediet. Temporal Factors in Mental Work: Effects of Interrupted Activities. Journal of Occupational and Organizational Psychology, 72, 163-185, 1999. 